\title{
Corela
}

Cognition, représentation, langage

HS-5 | 2006

Organisation des textes et cohérence des discours

\section{Relations de cohérence en discours : critères de reconnaissance, caractérisation et articulation cohésion-cohérence}

Francis Cornish

\section{OpenEdition}

Journals

Édition électronique

URL : http://journals.openedition.org/corela/1456

DOI : 10.4000/corela.1456

ISSN : $1638-573 X$

Éditeur

Cercle linguistique du Centre et de l'Ouest - CerLICO

Référence électronique

Francis Cornish, «Relations de cohérence en discours : critères de reconnaissance, caractérisation et articulation cohésion-cohérence », Corela [En ligne], HS-5 | 2006, mis en ligne le 27 octobre 2006, consulté le 01 mai 2019. URL : http://journals.openedition.org/corela/1456 ; DOI : 10.4000/ corela.1456

Ce document a été généré automatiquement le 1 mai 2019.

\section{(c) (1) (-)}

Corela - cognition, représentation, langage est mis à disposition selon les termes de la licence Creative Commons Attribution - Pas d'Utilisation Commerciale - Partage dans les Mêmes Conditions 4.0 International. 


\title{
Relations de cohérence en discours : critères de reconnaissance, caractérisation et articulation cohésion-cohérence
}

\author{
Francis Cornish
}

\section{NOTE DE L'AUTEUR}

Cet article est une version remaniée de la communication du même titre que j'ai présentée lors de la Journée Scientifique sur la Cohésion et la Cohérence au laboratoire CRISCO à l'Université de Caen, le 27 mai 2005. Je remercie Dominique Legallois et Anne Grobet de leur relecture attentive d'une version préliminaire, ainsi que Christine Pernet pour ses corrections de forme et de style.

\section{Introduction}

1 Cet article fait suite à la communication présentée à la Journée Scientifique "Unité(s) du texte" organisée par le laboratoire CRISCO à l'université de Caen le 6 décembre 2002 (voir Cornish, 2003a pour le texte de cette communication, et Cornish, 2006 pour une version révisée). Dans ce travail, j'avais cherché à mettre en évidence les interactions qu'il y a dans l'intégration du contenu d'une suite de phrases écrites, souvent non reliées par un connecteur explicite, comprise dans de courts textes d'articles de faits divers ou de résumés de films, entre d'une part, le choix d'une relation de cohérence et de l'autre, la résolution d'anaphores dans les phrases non initiales. J'avais surtout mis à contribution dans cette communication l'approche de J.R. Hobbs (1990: ch.5) dans la définition sémantique d'un certain nombre de relations de cohérence, et leurs connections intimes avec l'anaphore. Depuis, nous avons mis en place un projet inter-universitaire (Toulouse 
II et Strasbourg II) sous l'égide de l'Institut de Linguistique Française (ILF), qui étudie ces interactions en profondeur. ${ }^{1}$

Le présent article portera principalement sur les critères et les indices de reconnaissance d'un sous-ensemble de relations de cohérence (ou de discours). Ces relations permettent de dégager et d'intégrer la valeur énonciative et interactive, puis le contenu des propositions (syntaxiques) dans de courts textes écrits, souvent sans connecteur explicite. L'idée de ne retenir dans un premier temps que de courts textes de ce genre, se justifie par le fait que ce sont en premier lieu des textes réels (et non construits), conçus à l'origine comme tels par leur scripteur; et en second lieu, ils constituent des sortes de laboratoires en miniature, permettant de cerner plus aisément les relations discursives qui sont en jeu. Dans la mesure où l'on reste à l'intérieur d'un même genre (les articles de faits divers, ou bien des résumés de films), l'analyste peut donc contrôler les variables à l'œuvre, tout en bénéficiant des avantages fournis par l'existence d'un corpus de textes attestés. ${ }^{2}$

On s'attachera également à déterminer les unités de discours (tant globales que locales) impliquées dans cette intégration, puis leur statut d'unité indépendante (ou « noyau ») ou bien dépendante ("satellite»), et enfin le point d'attachement des unités dépendantes, satellites, par rapport à l'unité qu'elles étendent sémantiquement. Il s'agira plus largement de repérer les points d'articulation entre la cohésion du texte et la cohérence du discours qui peut lui être associée, en fonction du choix d'un contexte approprié. Mais pour commencer, nous aborderons surtout de façon critique l'approche des relations de cohérence développée par la Rhetorical Structure Theory (RST) de W.C. Mann et S.A. Thompson, modèle largement utilisé aujourd'hui en analyse de discours.

\section{Définitions et analyses de la RST : quelques problèmes}

4 Halliday et Hasan (1976) proposaient une approche purement intra-textuelle des moyens de réaliser la cohésion dans les textes en anglais ; l'ensemble des cinq types de procédures qu'ils retenaient était fondé à partir d'un élément présupposant à relier à un élément présupposé à l'intérieur d'un même texte (que les éléments en question se trouvent dans une même phrase ou bien dans des phrases distinctes). La cohésion du texte à l'œuvre était censée jaillir de ces mises en relation intra-textuelles. Les auteurs laissaient hors de propos tout ce qui relève du discours, au sens où j'entends ce terme (voir le Tableau 1 cidessous). De toute façon, Halliday considère les notions de texte et de discours comme étant quasi-identiques, équivalentes (voir Butler, 2003 : 303).

De façon symétrique, l'approche des relations de cohérence dans les textes écrits proposée par W.C. Mann et S.A. Thompson (1988) prend ses distances par rapport à tout ce qui est textuel, relevant donc de la forme linguistique, pour ne tenir compte dans les définitions et critères de reconnaissance que des intentions présumées du scripteur, et de l'effet que celui-ci entend produire en invoquant comme cadre cognitif pour la compréhension de ces intentions telle ou telle relation de cohérence (ou de discours). Pourtant, dans les représentations schématiques de la structure des textes qu'ils présentent, ce sont toujours des segments de texte qui sont représentés. Il est cependant clair que les deux dimensions, celles du texte et du discours (de même que le contexte, bien entendu!), doivent être prises en compte au même titre dans la mise à plat du 
fonctionnement des textes, en terme de leur structuration en unités ainsi que des relations à l'œuvre entre ces unités.

La distinction texte/discours peut se définir comme suit (voir aussi Widdowson, 2004 : ch.1) :

Tableau 1 : Rôles du texte, du discours et du contexte

\begin{tabular}{|c|c|c|}
\hline Texte & Discours & Contexte \\
\hline $\begin{array}{l}\text { La séquence connexe de } \\
\text { signes verbaux et de } \\
\text { signaux non verbaux en } \\
\text { fonction de laquelle le } \\
\text { discours est co- } \\
\text { construit par les } \\
\text { participants dans l'acte } \\
\text { de communication. }\end{array}$ & $\begin{array}{l}\text { Le produit de la séquence } \\
\text { hiérarchiséer et } \\
\text { contextuellement située } \\
\text { d'actes énonciatifs, } \\
\text { indexicaux, propositionnels, } \\
\text { et illocutoires effectuées } \\
\text { dans la poursuite d'un but } \\
\text { communicatif quelconque, } \\
\text { et intégrés dans un contexte } \\
\text { donné. }\end{array}$ & $\begin{array}{l}\text { Le contexte (grosso modo, le domaine } \\
\text { de référence d'un texte, et les } \\
\text { circonstances de sa situation } \\
\text { d'énonciation) est assujetti à un } \\
\text { processus continu de construction et } \\
\text { de révision au fur et à mesure que le } \\
\text { discours se déroule. C'est à travers } \\
\text { l'invocation d'un contexte approprié } \\
\text { (contexte qui est en partie déterminé } \\
\text { par la nature du co-texte à l'œuvre, } \\
\text { ainsi que par son genre) que l'auditeur } \\
\text { ou le lecteur peut convertir la } \\
\text { séquence connexe d'indices textuels } \\
\text { qui est le texte en discours. }\end{array}$ \\
\hline
\end{tabular}

Cornish, 2003b : 3, légèrement révisé

Le texte est la trace d'au moins un acte d'énonciation (qu'il soit réalisé en termes d'une trace verbale, linguistique, ou bien non-verbale - qui, elle, peut être gestuelle, sensoriperceptuelle ou prosodique). La notion de texte est proche de ce que Gumperz (1992 : 234) appelle "indices de contextualisation". Les partenaires de discours exploitent cette trace conjointement à l'invocation d'un contexte approprié, en termes cognitifs, afin de construire du discours.

7 Le discours, en revanche, renvoie au produit hiérarchiquement structuré, mentalement représenté, de la séquence d'actes énonciatifs, propositionnels, illocutoires et indexicaux que les participants effectuent au fur et à mesure que la communication se déroule. De telles séquences ont pour raison d'être la réalisation d'un but communicatif local et/ou global quelconque (voir Parisi \& Castelfranchi, 1977).

Le point crucial dans cette distinction est que le discours est une affaire (re)constructive, et donc hautement probabiliste: du point de vue de l'allocutaire, il ne s'agit nullement d'un simple décodage du texte afin d'arriver au message complet voulu par l'énonciateur. Le discours, donc, est à la fois un construit hiérarchisé et défaisable (une construction provisoire d'une interprétation située), alors que le texte est essentiellement linéaire cependant, dans la forme orale, des phénomènes paralinguistiques, non-verbaux peuvent co-apparaître simultanément avec le flux des signes et signaux verbaux. Le 'sens' ne réside pas entièrement "dans" le texte, il doit être construit par l'allocutaire (et l'énonciateur!) via le texte et un contexte approprié (cf. Coupland et al., 1991: 5). De toute manière, le texte est souvent, sinon toujours, à la fois incomplet et indéterminé par rapport au discours qui peut en être dérivé à l'aide d'un contexte. 


\subsection{Présentation de l'approche de Mann et Thompson (la RST)}

La RST se présente comme un cadre descriptif pour la caractérisation de la structure des textes, mettant en évidence leur structure hiérarchique. Il s'agit d'une méthode pour décrire les relations sémantiques existant entre les propositions («clauses») dans un texte, que ces relations soient signalées lexicalement ou grammaticalement. Elle prétend également pouvoir caractériser les "Relational Propositions ", qui sont des propositions (logiques) implicites inférées automatiquement dans le processus de compréhension et d'interprétation des textes (voir Mann \& Thompson, 1986).

\subsubsection{Les relations}

Leurs définitions comportent :

1. Des restrictions sur le Noyau

2. Des restrictions sur le Satellite

3. Des restrictions sur la combinaison du Noyau et du Satellite

4. L'effet produit.

11 Lors de l'analyse d'un texte donné, l'analyste doit faire des jugements sur le scripteur et sur le lecteur. Ces jugements sont par définition probabilistes et défaisables. Ce sont des jugements de plausibilité.

\subsubsection{Les schémas}

12 Les schémas sont des patrons abstraits qui consistent en un petit nombre d'empans de texte constituants, une spécification de la relation les reliant, et une indication de la façon dont certains empans (des noyaux) sont reliés à l'ensemble. Les schémas, définis en termes de relations, indiquent comment des empans de textes peuvent co-apparaitre. Conjointement aux conditions d'application des schémas, ils déterminent les structures RST des textes. La RST reconnaît cinq types de schémas (voir la Figure 1). Les courbes représentent les relations, et les lignes verticales l'identification de l'empan/des empans noyau(x). Les schémas pour les relations non indiquées dans la Figure suivent tous le patron simple représenté par la relation Circonstance : une seule relation avec noyau et satellite. Les noms des schémas sont identiques aux noms de la relation correspondante. La grande majorité des schémas ainsi que des applications de schémas suivent ce patron simple. 
Figure 1 : Exemples des 5 types de schémas

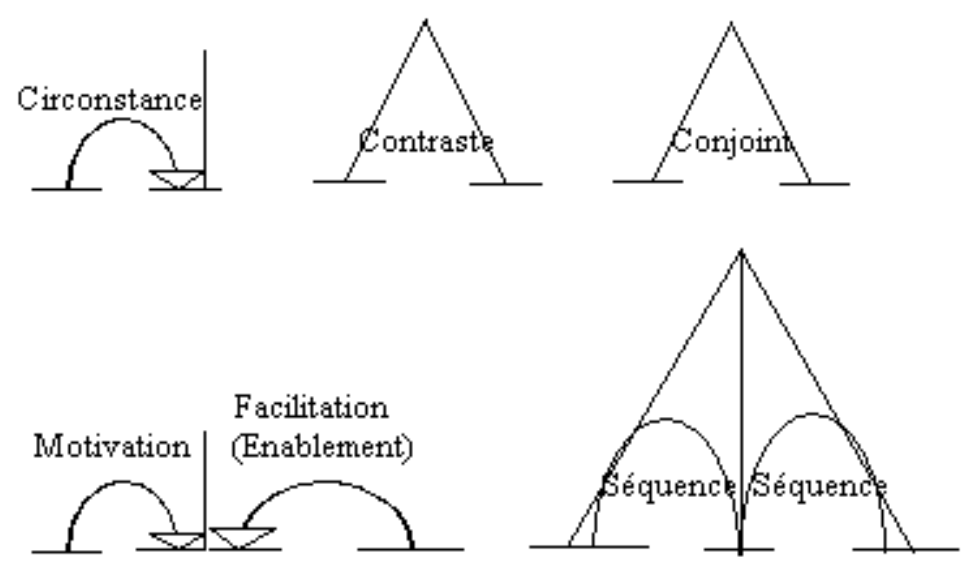

MANN ET THOMPSON, 1988 : FIgURE 1, P. 247

Les schémas multi-noyaux sont employés pour représenter un petit nombre de segments de texte, dans lesquels un autre patron d'organisation est employé au lieu d'une organisation autour d'un noyau unique. Le schéma Contraste a toujours exactement deux noyaux. Celui de Séquence en a un nombre indéfini, l'un pour chaque élément de la séquence, puis une relation de succession (suite) entre noyaux adjacents. Le schéma Conjoint a également un nombre indéfini de noyaux.

\subsubsection{Analyses structurelles et représentations des structures}

Première étape dans l'analyse d'un texte : le diviser en unités. La taille des unités est arbitraire, mais la division du texte en unités devra se baser sur une classification qui soit «neutre » par rapport à une théorie. ${ }^{3}$ Les unités dégagées devraient avoir une intégrité fonctionnelle indépendante. Pour les analystes RST, ces unités correspondent essentiellement (de façon minimale) à des propositions («clauses») - mais les sujets propositionnels et les relatives restrictives sont considérés comme faisant partie de leur proposition hôte, plutôt que comme des unités séparées.

Dans les représentations diagrammatiques en RST, les arcs, étiquetés par des noms de relations, relient des segments d'une structure pour lesquels une relation s'applique. Chaque ligne verticale descend de l'empan de texte en voie d'analyse au moyen d'une application de schéma jusqu'au noyau de l'application de schéma. Les chiffres représentent la séquence des unités non analysées de la structure. Pour certains textes, plus d'une seule analyse pourra être appropriée.

\subsection{Les relations et leur définition}

La RST propose une trentaine de relations de cohérence, avec leurs « définitions ». Cellesci ne sont pas basées sur les signaux morphologiques ou syntaxiques. La reconnaissance de la relation repose toujours uniquement sur des jugements fonctionnels et sémantiques. Les auteurs justifient cette position en observant, par exemple, que la reconnaissance de la relation Condition ne dépend pas de la présence de si.

Premier exemple de définitions, la relation Evidence ( Indice»), qui fait intervenir l'attitude du lecteur envers le noyau (donc, la proposition logique associée à la «clause » 
noyau). L'intention derrière l'emploi d'un satellite de type Evidence serait d'augmenter la croyance du lecteur en la proposition noyau. Les définitions des relations se font sous forme d'une série de critères de bonne formation. ${ }^{4}$ Exemple $(« \mathrm{~N} »=$ noyau; $" \mathrm{~S}$ » = satellite $" ; ~ « \mathrm{~L} »=$ lecteur ; $" \mathrm{Sc} »=$ scripteur). Pour la comparaison, je donne immédiatement après, la définition que j'ai formulée en termes du modèle proposé dans le chapitre 5 de Hobbs (1990) :

- Evidence (« Indice »)

- Contraintes sur N : L pourra ne pas croire en $\mathrm{N}$ à un degré suffisant pour Sc

- Contraintes sur $S: L$ croit $S$ ou le trouve crédible

- Contraintes sur la combinaison $N+S$ : La compréhension de S par L augmente sa croyance en $N$

- L'effet : La croyance de L en $\mathrm{N}$ est effectivement augmentée

- Lieu de l'effet : N (Mann et Thompson, $1988: 251)$

- Définition FC d'après l'approche de Hobbs (1990: ch. 5)

18 1) Inférer $P$ à partir de l'assertion de $S^{\circ}$ et de $S^{1}$ (essentiellement la relation d'Élaboration : voir la définition complète plus bas) et 2) interpréter $S^{1}$ comme rendant plus convaincante l'hypothèse de Sc correspondant à l'assertion de $\mathrm{S}^{\circ}$

19 L'exemple de cette relation donné par Mann et Thompson est celui-ci, où le scripteur est en train de faire la louange d'un programme de calcul des revenus provenant du Fisc américain :

(1) 1 . The programme as published for calendar year 1980 really works.

'Le système tel qu'il est publié pour l'année civile 1980 marche vraiment'

2. In only a few minutes, I entered all the figures from my 1980 tax return

'En quelques minutes seulement, j'ai entré tous les chiffres de ma déclaration de $1980 . . . '$

3. and got a result which agreed with my hand calculation to the penny.

'...et ai obtenu un résultat qui s'accordait avec mon calcul manuel au penny près'

(Mann et Thompson, $1988: 251$ )

Les unités $2+3$ ici entretiennent une relation de type Evidence par rapport à l'unité 1, dans le sens où elles sont données afin d'augmenter la croyance du lecteur en l'affirmation exprimée dans l'unité 1 . La coordination signalée par le connecteur and indique que les propositions 2 et 3 font partie d'une seule et même unité (un satellite, donc ; cf. aussi Blakemore, 2002).

Fig. 2 : Diagramme RST pour le texte (1)

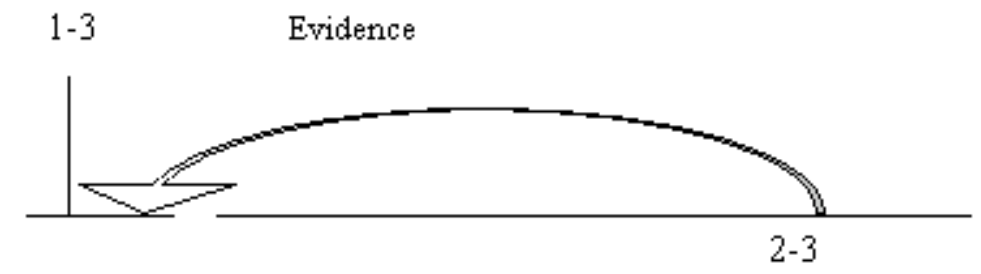

MANN ET THOMPSON, 1988 : FIg. 2, P. 251

Une autre paire de relations est représentée par Antithèse et Concession, qui partagent la propriété suivante : l'effet désiré consiste à faire en sorte que L ait une attitude positive 
par rapport à N. Elles diffèrent en ce que l'Antithèse est un sous-type de Contraste, alors que la Concession ne l'est pas.

- Antithèse

- Contraintes sur $N$ : Sc a une attitude positive par rapport à la situation présentée dans $\mathrm{N}$

- Contraintes sur $S$ : Aucune

- Contraintes sur la combinaison $N+S$ : les situations présentées dans $\mathrm{N}$ et $\mathrm{S}$ sont en opposition (cf. CONTRASTE, sc. Elles sont (a) comprises comme les mêmes à beaucoup d'égards, (b) comprises comme différant dans quelques aspects et (c) comparées par rapport à une ou plus d'une de ces différences); vu l'incompatibilité qui est inhérente au contraste, on ne peut avoir une attitude positive par rapport aux deux situations présentées dans $\mathrm{N}$ et dans $\mathrm{S}$; comprendre $\mathrm{S}$ ainsi que l'incompatibilité entre les situations présentées dans $\mathrm{N}$ et dans $\mathrm{S}$ augmente l'attitude positive de $\mathrm{L}$ vis-à-vis de la situation présentée dans $\mathrm{N}$

- L'effet : L'attitude positive de L vis-à-vis de $\mathrm{N}$ est augmentée

- Lieu de l'effet : N (Mann et Thompson, $1988: 253$ )

Voici, à titre de comparaison, la définition donnée de la relation Contraste, suivie de celles proposées par Hobbs (1990: ch. 5) :

- Contraste

- Contraintes sur N : multi-noyaux

- Contraintes sur la combinaison des noyaux: pas plus de deux noyaux; les situations présentées dans ces deux noyaux sont (a) comprises comme les mêmes à beaucoup d'égards, (b) comprises comme différant par quelques aspects et (c) comparées par rapport à l'une ou plus d'une de ces différences

- L'effet : L reconnaît la comparabilité et les différences fournies par la comparaison effectuée

- Lieu de l'effet : noyaux multiples (Mann et Thompson, $1988: 277$ )

\section{Définitions proposées par Hobbs (1990 : 99)}

1) Inférer $p(a)$ à partir de l'assertion de $S^{\circ}$ et $-p(b)$ de l'assertion de $S^{1}$, où $a$ et $b$ sont semblables. 2) Inférer $p(a)$ à partir de l'assertion de $S^{\circ}$ et $p(b)$ de l'assertion de $S^{1}$, où il existe une propriété $q$ telle que $q(a)$ et $\sim q(b)$.

La différence essentielle par rapport à la définition de la relation Antithèse, c'est qu'aucune mention n'est faite dans la définition de Contraste de l'augmentation de l'attitude positive de L vis-à-vis de la situation présentée par N (l'un ou l'autre des deux), ainsi que le fait qu'Antithèse comporte un $\mathrm{N}$ et un $\mathrm{S}$, alors que Contraste ne possède que deux noyaux. La première relation est donc conçue comme étant asymétrique, et la seconde comme symétrique.

Exemple de la relation Contraste (tiré de Scientific American) :

(2) 1 . Animals heal,...

'Les animaux guérissent,...'

2. but trees compartmentalize.

'mais les arbres s'organisent en compartimentant leurs systèmes de défense'

3. They endure a lifetime of injury and infection

'Ils endurent pendant toute une vie des blessures et des infections...'

4. by setting boundaries that resist the spread of the invading micro-organisms.

'...en mettant en place des frontières qui résistent à la propagation des micro-

organismes envahisseurs' [la division en unités, ainsi que leur numérotation, sont de moi]

(Mann et Thompson, $1988: 277$ )

On pourra analyser le texte (2) comme ayant un segment «noyau » composé des deux noyaux qui correspondent aux deux coordonnées de 1 et de 2, puis d'un "satellite » 
complexe composé du noyau 3 étendu par le satellite 4. La relation qui s'impose, intuitivement, pour relier (2.3) et (2.4) est celle de Moyen, mais cette relation ne semble pas être reconnue par Mann \& Thompson. ${ }^{5}$ Le «satellite» composé figuré par la composition de 3 et de 4 vient s'appuyer sur 2 uniquement, de par la relation anaphorique qu'entretient le pronom sujet they de 3 avec 'les arbres' évoqués dans 2. Il y a d'ailleurs une relation de topicalité vis-à-vis de ce seul référent contractée par le reste de ce court texte.

Cet exemple montre l'effet dynamique de ré-analyse d'un segment de texte qui peut provenir de l'effort d'intégration de segments ultérieurs du texte concerné : car au niveau de la prise en compte de la coordonnée en $1+2$, les deux propositions sont effectivement symétriques, correspondant à deux noyaux d'égale importance. Mais suite à l'intégration du « satellite » composé de la composition de 3 et de 4 uniquement avec le second de ces deux noyaux (intégration dirigée par la relation anaphorique visée ainsi que par la relation d'à-propos impliquée), ce second noyau en vient à assumer une dominance au niveau du discours par rapport à 1 . Cette situation ne semble pas être prévue par les auteurs, qui donnent tout de même cet exemple en illustration de leur méthode d'analyse des textes! (Il est censé illustrer leur relation Contraste). La relation en fonction de laquelle $3+4$ serait intégré avec 2 est celle d'Evidence ( Indice ») : 3+4 fournit une preuve du bien-fondé de l'assertion faite via l'énonciation de 2 en contradiction avec celle de 1 . Le connecteur after all 'après tout', ou bien indeed 'en effet' pourra naturellement être intercalé entre 2 et 3 ici.

Une autre analyse, encore, qui mettrait en évidence le caractère asymétrique des deux propositions (structure «satellite-noyau ») serait de proposer que 1 et 2 sont reliées en fonction de la relation Concession, avec 1 comme satellite et 2 comme noyau. Selon cette analyse, le connecteur although 'bien que' pourra être préfixé à 1, et but supprimé.

Figure 3 : Diagramme de la RS du texte (2) (FC)

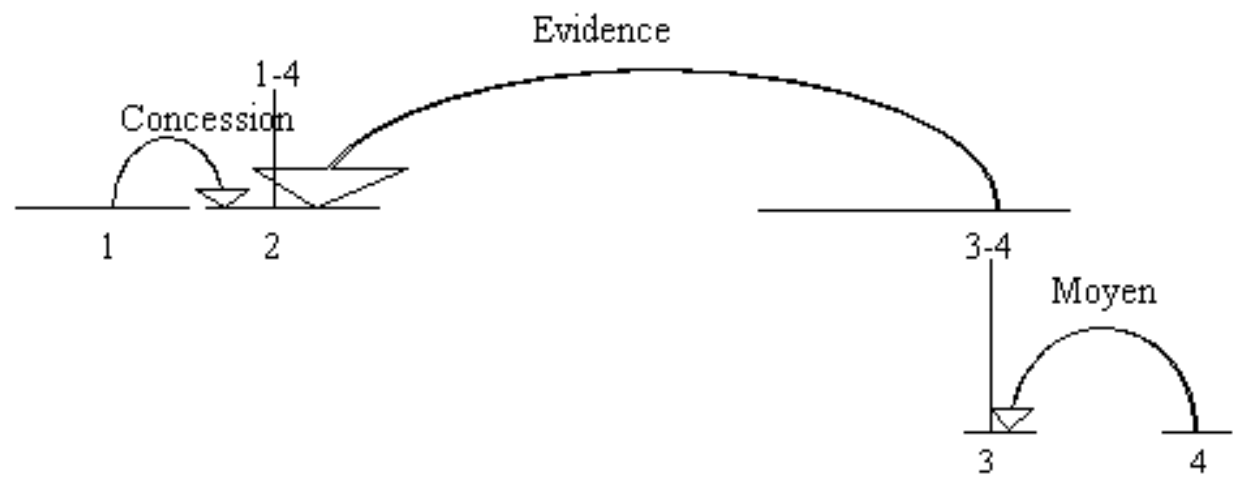

La relation de Concession est définie ainsi :

\section{- Concession}

- Contraintes sur $N$ : Sc a une attitude positive vis-à-vis de la situation présentée dans $\mathrm{N}$

- Contraintes sur $S: S c$ ne prétend pas que la situation présentée dans $S$ ne tient pas

- Contraintes sur la combinaison $N+S$ : Sc reconnaît l'existence d'une incompatibilité potentielle ou apparente entre les situations présentées dans $\mathrm{N}$ et dans $\mathrm{S} ; \mathrm{Sc}$ considère que les situations présentées dans $\mathrm{N}$ et dans $\mathrm{S}$ sont en fait compatibles; le fait de reconnaître la compatibilité entre les situations présentées dans $\mathrm{N}$ et dans $\mathrm{S}$ augmente l'attitude positive de L vis-à-vis de la situation présentée dans $\mathrm{N}$ 
- L'effet : L'attitude positive de L vis-à-vis de la situation présentée dans $\mathrm{N}$ est augmentée

- Lieu de l'effet : N et S (Mann et Thompson, 1988 : 245-255)

- Définition à la Hobbs (1990) (FC) : Inférer $p(a)$ à partir de l'assertion de $S^{\circ}$ et $p(b)$ de l'assertion de $S^{1}$, où il existe une propriété $q$ telle que $q(a)$ et $\sim q(b)\left(=2^{\text {ème }}\right.$ alternative de sa définition de la relation Contraste). Cependant, le locuteur/le scripteur considère que $q(b)$ est néanmoins vraie, étant donné $q(a)$.

Exemple:

(3) Dioxin

1. Concern that this material is harmful to health or the environment may be misplaced.

'L'inquiétude quant à la nocivité de cette substance pour la santé ou l'environnement pourrait être mal fondée'

2. Although it is toxic to certain animals, 'Bien qu'elle soit toxique pour certains animaux,'

3. evidence is lacking that it has any serious long-term effect on human beings.

'les preuves manquent pour dire qu'elle produit un effet grave à long terme sur les humains'

(Mann et Thompson, $1988: 255$ )

Figure 4 : Diagramme RST pour le texte (3)

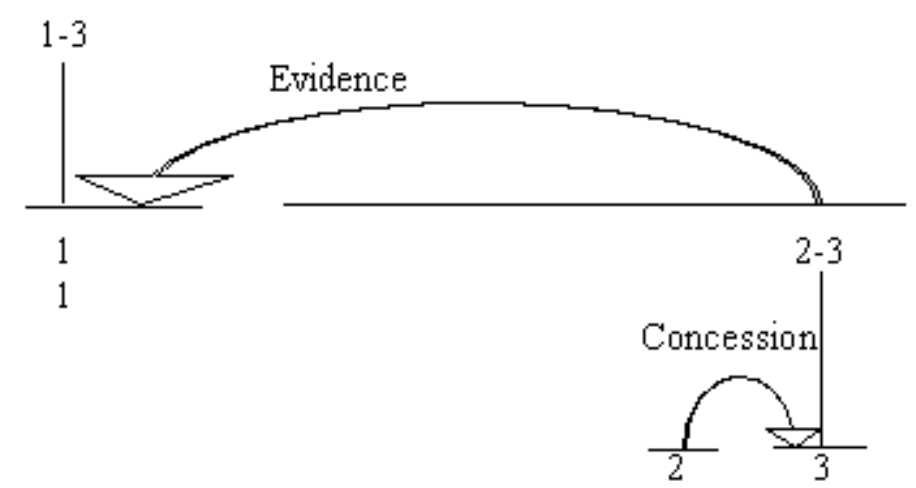

MANN ET THOMPSON, 1988 : FIg. 5, P. 255 - LÉgÈREMENT ADAPTÉ

J'ai remplacé la mention "Élaboration" reliant $2+3$ à 1 dans la Fig. 4 par celle d' «Evidence ", car 2+3 fournit bien une preuve du bien-fondé de l'assertion dans 1 que l'inquiétude en question est mal placée. ${ }^{6}$ Si l'on pose que plus d'une RC soit possible à la fois pour intégrer deux unités, dans ce cas, c'est la relation la plus «forte», sémantiquement, qui prendra le pas sur la relation plus faible. La relation Evidence ("Indice ») est sémantiquement plus forte qu'Elaboration, car elle comprend la même structure de base que celle-ci (les deux propositions syntaxiques concernées doivent pouvoir évoquer la même proposition logique, et la seconde doit apporter au moins une information supplémentaire par rapport à la première $^{7}$; mais pour que la relation Evidence soit pertinente, la seconde doit pouvoir s'interpréter comme rendant l'assertion de la première plus convaincante). ${ }^{8}$ Alors qu' Elaboration est une simple relation « sémantique », Evidence, elle, est " pragmatique » (voir le Tableau 2a dans la sous-section 2.3 supra). Cornish (2006) donne plusieurs autres exemples de cas où plus d'une relation est invoquée en même temps pour intégrer deux unités.

Voici la définition donnée pour la relation Élaboration ${ }^{9}$ :

\section{- Élaboration}


- Contraintes sur la combinaison $N+S: S$ présente des informations supplémentaires vis-à-vis de la situation ou de quelqu'aspect du thème présenté(e) dans $\mathrm{N}$ ou bien qui est accessible par inférence dans $\mathrm{N}$ au moyen d'une ou de plusieurs des manières énumérées ci-dessous. Dans cette liste, si $\mathrm{N}$ présente le premier membre d'une paire quelconque, alors $\mathrm{S}$ comprend le second :

1. ensemble : membre

2. abstraction : exemplaire (« instance »)

3. tout : partie

4. processus : étape

5. objet : attribut

6. généralisation : élément spécifique

- L'effet: L reconnaît la situation présentée dans S comme fournissant des informations supplémentaires au sujet de N. L identifie l'élément de la thématique pour lequel un détail est fourni.

- Lieu de l'effet : $\mathrm{N}$ et S (Mann et Thompson, $1988: 273$ )

\section{Définition proposée par Hobbs (1990 : 95), étendue par moi-même}

Inférer la même proposition $P$ à partir de l'assertion de $S^{\circ}$ et de $S^{1}$. (J'ajouterais à cette définition la condition (essentielle pour la relation Elaboration) que $\mathrm{S}^{1}$, la proposition "élaborante", fournisse à la proposition inférée commune des informations supplémentaires, et que $\mathrm{e}^{1} \subseteq \mathrm{e}^{0}$ (l'événement principal évoqué par $\mathrm{S}^{1}\left(\mathrm{e}^{1}\right)$ serait à interpréter comme constituant une partie essentielle de celui dénoté par $\left.\mathrm{S}^{\circ}\left(\mathrm{e}^{\circ}\right)\right)$. Si ces conditions ne sont pas appliquées, alors la définition que propose Hobbs reviendrait à ne caractériser qu'une simple relation de Paraphrase).

Comme le note Hobbs (p. 95), cette définition est la relation Parallèle lorsque les entités similaires ai et bi dans chaque schéma propositionnel ouvert qui constitue la structure de cette relation, sont identiques, pour tout $i$. D'après cette définition, il est évident que $2+3$ dans (3) fournit une " élaboration » du noyau 1, mais il est tout aussi évident qu'il s'agit également d'une preuve du bien-fondé de l'assertion de 1. Comme le dit Hobbs (1990: ch. 5), il est clair que plus d'une relation peut coexister à la fois, entre deux segments de discours (voir aussi Asher \& Lascarides, 2003). Cependant, cette possibilité est expressément exclue par la RST ${ }^{10}$.

Un mot à présent sur les formulations des définitions présentées ci-dessus pour les mêmes relations par la RST et par Hobbs (à savoir, Evidence, Contraste, Concession et Elaboration). La définition proposée par Mann et Thompson pour Evidence insiste uniquement sur le fait que la compréhension du satellite par le lecteur vise à augmenter sa croyance en la proposition (ou l'assertion de la proposition) exprimée par le Noyau, du point de vue du scripteur. Celle de Hobbs (c'est-à-dire, celle que j'ai formulée sur la base du type de définitions proposées par lui) indique en plus que les deux unités doivent pouvoir évoquer la même proposition (comme dans la relation Elaboration, et d'ailleurs dans l'ensemble des relations d'« expansion» que propose Hobbs). Quant à Contraste, les deux définitions sont quasiment identiques. La formulation de Hobbs a l'avantage d'être plus précise, car plus formelle. Dans le cas de Concession, les définitions sont sensiblement les mêmes, toutes les deux reconnaissant qu'un ingrédient de la relation Contraste y est impliqué. La définition RST ajoute l'indication d'ordre « argumentatif » que la concession que le satellite soit néanmoins vrai est destinée à augmenter la crédibilité du noyau aux yeux du lecteur. Enfin, dans le cas d'Elaboration, les deux définitions (celle de Hobbs révisée par moi-même) indiquent le nécessaire ajout d'informations supplémentaires par 
l'unité élaborante vis-à-vis de l'unité élaborée et la relation "partie-tout» qui les caractérise, Hobbs soulignant l'inférence d'une proposition commune par les deux unités.

\subsection{Principes de regroupement des relations}

Une façon de regrouper les relations retenues serait de faire une distinction entre les aspects 'thématiques' et 'présentationnels' de la structure des textes. Ainsi, des relations comme celle de Cause intentionnelle expriment certains aspects de la thématique du texte. Celle-ci met en rapport deux empans de texte s'ils sont compris comme étant reliés de façon causale en termes du contenu thématique. D'autres, comme celle de Justification, ne sont employées que pour faciliter la présentation du texte elle-même. La Justification ne relie deux empans de texte que si l'un d'entre eux est jugé de nature à augmenter l'acceptation par le lecteur de l'autre. L'indicateur le plus net de l'existence de l'un ou de l'autre type de relations est celui de l'effet que telle relation a (ou est censé avoir) sur le lecteur. Les relations "thématiques» sont celles dont l'effet visé est que le lecteur identifie la relation en question. Par contre, les relations « présentationnelles » sont celles dont l'effet souhaité est d'augmenter telle ou telle disposition chez le lecteur, comme le désir d'agir ou le degré de son attitude positive envers, ou croyance en, ou encore acceptation de la proposition noyau. Le Tableau 2 présente cette classification.

Tableau 2. Classification des relations sur la base 'thématique' vs. 'présentationnel

\begin{tabular}{|l|l|}
\hline Thématique & Présentationnel \\
\hline Elaboration & \\
Circonstance & \\
Problème-Solution & \\
Cause intentionnelle & \\
Résultat intentionnel & Motivation \\
Cause non-intentionnelle & Antithèse \\
Résultat non-intentionnel & Arrière-plan \\
But & Facilitation \\
Condition & Evidence (« indice ») \\
Interprétation & Justification \\
Evaluation & Concession \\
Reformulation & \\
Résumé & \\
Séquence & \\
Contraste & \\
\hline
\end{tabular}

Tableau 3 dans Mann \& Thompson, 1988 : 257

S'agissant de la catégorie 'Présentationnel', suivant Roulet (2002), Smith (2003) et d'autres encore, on pourra utilement la scinder en deux sous-catégories, selon que la vocation de la relation en question est de signaler la structure ou l'organisation du texte, ou de remplir une fonction plus pragmatique (faisant ainsi intervenir la relation interlocutive 
entre scripteur et lecteur, ou exprimant la visée communicative de Sc vis-à-vis de L). La catégorie 'thématique' proposée par Mann et Thompson dans le Tableau 2 correspondrait à la rubrique 'sémantique' chez ces auteurs. Voici donc une variante du Tableau 2 (Tableau 2a) qui manifeste ces trois catégories de fonctionnalité des relations de cohérence. Avec la présence d'une catégorie plus particulièrement textuelle, j'ai pensé qu'il serait préférable de ranger les relations Reformulation et Résumé (respectivement « Restatement " et "Summary », chez Mann et Thompson) dans cette catégorie. C'est ce que propose également Smith $(2003: 264)$, en appelant cette dernière catégorie "Rhétorique " (elle inclut également dans cette catégorie la relation «Parallèle », qu'on retrouve chez Hobbs,1990, mais non chez Mann \& Thompson).

Tableau 2a. Classification des relations en fonction des critères 'sémantique', 'pragmatique' et 'textuel'

\begin{tabular}{|l|l|l|}
\hline Sémantique & Pragmatique & Textuel \\
\hline Elaboration & & \\
Circonstance & & \\
Problème-Solution & & \\
Cause intentionnelle & Motivation & \\
Résultat intentionnel & Antithèse & Arrière-plan \\
Cause non-intentionnelle & Facilitation & Reformulation \\
Résultat non-intentionnel & Evidence (« indice ») & Résumé \\
But & Justification & \\
Condition & Concession & \\
Interprétation & & \\
Evaluation & & \\
Séquence & & \\
Contraste & & \\
\hline
\end{tabular}

\section{Critères de reconnaissance des Relations de Cohérence : indices pertinents}

$34 \mathrm{Au}$ cours de notre présentation de l'application de l'analyse RST à des textes courts, ${ }^{11}$ plusieurs problèmes sont apparus. Tout d'abord, les définitions proposées des différentes relations de cohérence retenues ne sont pas univoques: dans l'exemple (2), nous avons vu que plutôt que la relation Contraste, comme l'indiquaient les auteurs, c'est celle de Concession qui devrait être invoquée pour relier les propositions 1 et 2, cette dernière étant le noyau sur lequel s'applique le satellite complexe figuré par les propositions $3+4$ reliées l'une à l'autre via la relation « Moyen ». Dans ce cas, conformément à la définition de la relation Concession, la proposition 1 est satellite (donc dans une relation de dépendance) et la 2 le noyau. C'est seulement dans un deuxième temps, suite à 
l'attachement de 3+4 à 2 grâce à l'anaphore réalisée par le pronom sujet they, que cette (ré)analyse de la relation reliant 1 et 2 peut se faire.

Dans l'exemple (3), exemple proposé par les auteurs pour illustrer la relation Concession (relation signalée explicitement ici pour relier les propositions 2 et 3 grâce à la présence $\mathrm{du}$ connecteur although 'bien que'), la relation (dominante, en tout cas) reliant les propositions 1 et 2+3 n'est pas tant celle d'Elaboration, comme le prétendent les auteurs, mais plutôt Evidence («Indice»). D'une part, il est possible, comme nous l'avons vu, que plus d'une relation de cohérence s'impose à la fois; et de l'autre, la relation d'Elaboration est fort générale (nous avons vu avec Hobbs, 1990: ch. 5 dans Cornish, 2003a ; 2006, qu'elle sous-tend en fait toutes les relations qu'il regroupe sous la rubrique «Relations d'Expansion »: Parallèle, Contraste, Généralisation, Exemplification, relations auxquelles nous avons ajouté celle d'Evidence).

Dans deux des trois exemples proposés par Mann \& Thompson (1988) pour illustrer l'application de certaines de leurs relations de cohérence, nous avons donc vu que les analyses qui s'imposent ne correspondent pas à celles qu'ils indiquent. Ceci est sans doute dû au vague de leurs définitions, formulées quasi uniquement en terme des intentions imputées au scripteur vis-à-vis de son lecteur, et non en terme des indices linguistiques présents dans les textes. ${ }^{12}$ Concernant ceux-ci, il est clair que la conjonction and préfixant la proposition 3 dans l'exemple (1) signale que 2 et 3 sont reliées en une proposition conjointe en vertu de la relation Parallèle; que la proposition gérondive introduite par la préposition by en 4 dans l'exemple (2) signale sa subordination syntaxique ainsi que sémantique par rapport à la 3 (la relation Moyen étant indiquée pour les relier, en vertu $\mathrm{du}$ sens de la préposition en question); et que le pronom sujet they dans la proposition 3 conjointement à ce qui est prédiqué de son référent par le reste de cette proposition, oriente le lecteur vers un attachement de ce satellite complexe à la proposition $2 .{ }^{13} \mathrm{Par}$ contre, le sens instructionnel correspondant à la conjonction adversative but qui coordonne les propositions 1 et 2 de cet exemple n'est pas univoque : sa valeur purement oppositive n'est pas de mise dans ce contexte, car l'attachement du satellite complexe qu'est la combinaison de 3 et de 4 à la proposition 2 (de par l'anaphore) attribue une proéminence discursive plus élevée à la proposition 2 par rapport à la 1, ce qui favorise l'autre valeur possible de but, celle de marquer la concession. L'équipollence des deux propositions nécessaire pour l'attribution de la relation Contraste n'est donc pas assurée ici.

Dans l'exemple (3), la présence de deux occurrences du pronom it dans chacune des propositions 2 et 3 reliées en satellite complexe grâce au connecteur concessif although, relie à son tour ces deux propositions à la 1, en vertu de l'anaphore ainsi signalée avec this material qui y figure (et qui est lui-même en relation d'anaphore démonstrative avec le référent du titre ('Dioxin')) - ce qui l'érige en macro-topique de ce discours : la présence en tant que titre du texte l'indique déjà, d'ailleurs.

Dans le cas des propositions non reliées explicitement via un connecteur dans ces exemples, nous avons vu que l'insertion d'un connecteur de tel ou tel type permet de choisir telle ou telle relation de cohérence pour assurer leur intégration (cf. le test proposé par Knott et Sanders, 1998) : l'un des connecteurs after all ou indeed pourrait être inséré entre les propositions 1 et 2 dans l'exemple (1) sans affecter le sens de leur intégration, et de même entre les propositions 2 et 3 dans (2). Indeed aurait pu également apparaître devant Although à l'initiale de la p2 dans (3). Dans tous ces cas, le caractère acceptable de ces insertions indique fortement la pertinence de la relation Evidence permettant d'intégrer 
les propositions, ou la structure propositionnelle complexe et la proposition qui la précède. Entre la p1 et la p2 dans (2), le connecteur concessif although aurait pu remplacer la conjonction but, indiquant ainsi la pertinence de la relation Concession.

Il y a donc plusieurs types d'indices linguistiques permettant de déterminer la pertinence d'application de telle ou telle relation de cohérence. Cependant, ces indices ne sont pas dans un rapport biunivoque avec telle ou telle relation de cohérence ${ }^{14}$ pourtant, la combinaison d'indices de types différents peut bien permettre de conclure à l'applicabilité d'une relation quelconque. On l'a vu dans l'exemple (2), où la relation d'anaphore entre la p3 et la p2 (pronom they), conjointement à la possibilité de remplacer la conjonction but entre la $\mathrm{p} 1$ et la $\mathrm{p} 2$ par although, suggèrent fortement la RC Concession comme moyen de les intégrer, plutôt que Contraste.

D'autres indices sont les relations temporelles existant entre propositions : temps du verbe, adverbiaux temporels; puis les relations lexicales existant entre les prédicateurs des propositions à relier (relations d'hyponymie, de synonymie, de méronymie, d'antonymie ou la relation converse); l'Aktionsart des prédicateurs verbaux des deux propositions à relier : statuts d'état, d'activité, d'achèvement ou d'accomplissement, et enfin (mais la liste n'est pas exhaustive), la structure événementielle ou « aspectuelle» des deux propositions à l'œuvre dans leur ensemble. Les voici donc (Tableau 3) :

Tableau 3 : Indices de reconnaissance des relations de cohérence

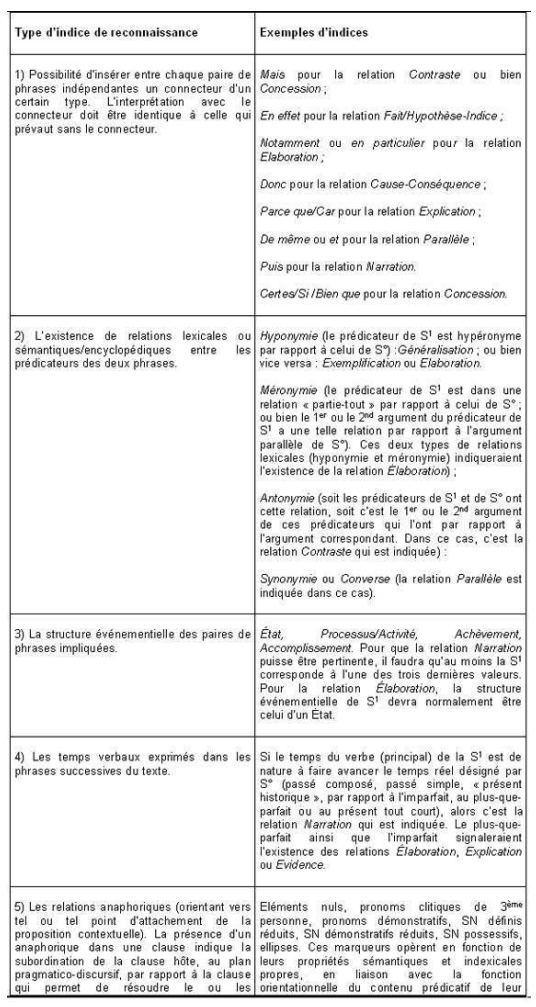

41 A ces critères devront s'ajouter la structure topicale des textes, qui interagit de façon centrale avec le choix de différents types de relations de cohérence pour intégrer les propositions inférables des clauses d'un texte, ainsi que la ponctuation qui les sépare (les textes du corpus étudié ici étant tous écrits). Aucun de ces critères, pris individuellement, ne serait suffisant pour fonder l'existence de telle ou telle relation de cohérence : c'est 
leur interaction plutôt qui permettrait éventuellement de le faire. Voici un exemple d'un résumé de film en illustration :

(4) « L LîlLE DE ROBINSON Téléfilm (...)

$\mathrm{S}_{\mathrm{EV}}^{\circ}{ }^{15} \mathrm{Au}$ XVIIIè siècle, le propriétaire d'une plantation s'oppose, $\mathrm{S}_{\mathrm{EV}}^{1}$ lors d'une

traversée maritime, au capitaine du bateau. $\mathrm{S}_{\mathrm{EV}}^{2} \mathrm{Il}$ est abandonné sur une île. » Le

Monde Radio-Télévision 22-28/12/03, p. 8.

La $\mathrm{S}_{\mathrm{EV}}^{\circ}$ de ce texte présente la situation de base qui forme la trame du film: le temps présent est un présent de narration (ceci est en partie du à l'Aktionsart du verbe s'opposer, et du SP événementiel en apposition lors d'une traversée maritime). Ce SP, de par la nominalisée qu'il contient, correspond à une unité de discours, marquée à la fois par la préposition temporelle lors (de) et par l'Aktionsart d'accomplissement représentée dans le $\mathrm{N}^{\prime}$ traversée maritime; ainsi, cette unité serait intégrée avec la $\mathrm{S}^{\circ}$ en terme de la relation Circonstance. Le passif du verbe abandonner de la $\mathrm{S}^{1}$ qui suit est compris comme faisant avancer l'action, mettant l'accent sur le résultat d'une action précédente. Le contenu de cette seconde phrase indépendante serait intégré à celui de l'unité composée $\left[\mathrm{S}^{\circ}+\mathrm{S}^{1}\right] \mathrm{de}$ façon minimale en vertu d'une relation de Résultat intentionnel, ce en fonction de notre connaissance du monde (surtout des mœurs maritimes de l'époque) : à savoir, que le capitaine d'un bateau a en principe le droit de régir le comportement des personnes à bord son navire. Etant donné l'existence d'une dispute entre celui-ci et un passager sur son bateau ('le propriétaire d'une plantation') évoquée par la $\mathrm{S}^{\circ}$, conjointement au pouvoir du capitaine sur les passagers à bord de son bateau, il est plus probable que ce serait le propriétaire de la plantation que le capitaine du bateau qui sera " abandonné sur une île ", suite à cette altercation. ${ }^{16}$ Pour ce qui concerne le test de l'insertion de tel ou tel type de connecteur entre les phrases à relier, dans (4), nous pouvons proposer que le connecteur de ce fait marquant la relation de Résultat, aurait pu être prefixé à la $\mathrm{S}^{2}$. Comme me le fait remarquer Dominique Legallois, cette relation serait chapeautée ici par celle de Problème-Solution, étant donné que $\mathrm{S}^{\circ}$ fait état d'une situation conflictuelle (... s'oppose...), donc "problématique », situation à laquelle l'abandon du propriétaire de la plantation sur une île pourrait constituer une "solution", aux yeux du capitaine. Le même raisonnement concernant la résolution du pronom anaphorique il sujet de la $\mathrm{S}^{2}$ pour fonder la pertinence de Résultat intentionnel s'appliquerait dans ce cas (voir plus bas).

La mise en place des relations Résultat intentionnel et Problème-Solution afin d'intégrer les deux propositions exige donc que le pronom sujet il soit résolu en ciblant le propriétaire de la plantation, plutôt que le capitaine. En principe (hors contexte particulier), cependant, ce pronom masculin singulier pourrait être interprété comme coréférant au 'capitaine', voire au 'bateau'. Après tout, et un bateau et un capitaine de navire peuvent fort bien « être abandonnés sur une île ». Mais dans l'un comme l'autre de ces deux cas, les relations Résultat intentionnel et Problème-Solution n'auraient que peu de justification comme moyens d'intégrer les deux propositions à l'œuvre ici. La Fig. 5 représenterait la structure du texte (4) d'après la RST, et la Fig. 5a selon l'approche de Hobbs (1990). À noter que c'est la représentation hobbsienne qui montre de façon plus explicite la hiérarchisation des rattachements entre les unités en jeu ici (les symboles $\mathrm{S}^{\mathrm{n}}$ en gras dans cette dernière Figure représentent les unités dominantes). Comme Mann et Thompson excluent la possibilité que deux relations s'appliquent pour relier deux unités simultanément, les deux relations en question sont présentées ici comme alternativement pertinentes pour intégrer l'unité 3 à l'unité composée $2+1$ dans la Figure 5 ; mais comme Hobbs, lui, l'admet, Problème-Solution est superposée à Cause-Conséquence (l'équivalent 
hobbsien de Résultat intentionnel chez Mann et Thompson) dans la Figure 5a, sa précédence indiquant qu'elle est dominante par rapport à celle-ci (ce système de représentation est de mon ressort).

Figure 5 : Diagramme RST pour le texte (4) (FC)

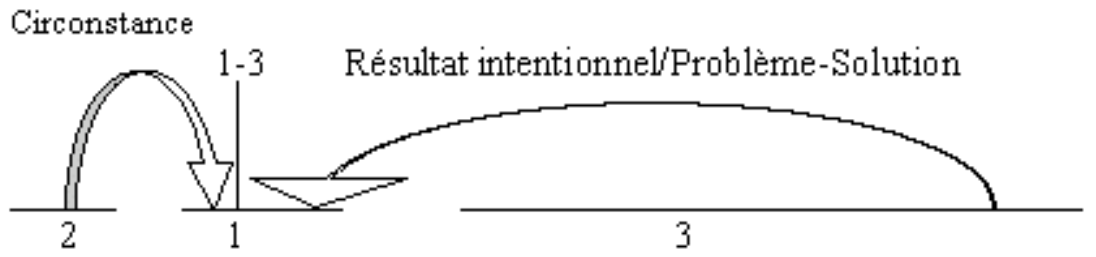

Figure 5a : Représentation d'après Hobbs (1990) de la structure de discours associée à (4)

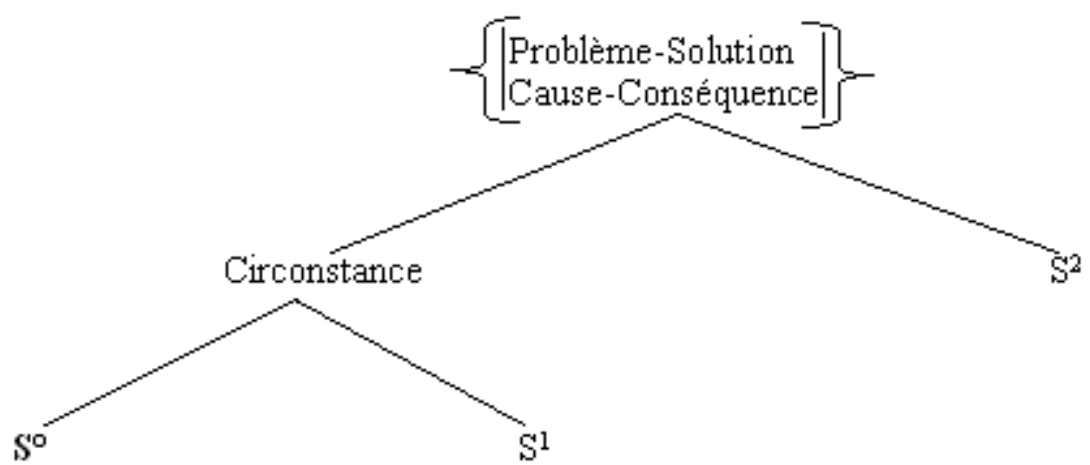

Nous venons de voir avec les exemples présentés jusqu'ici le rôle de plusieurs types d'indice de reconnaissance dans le choix de la pertinence de telle ou telle relation de cohérence: le jeu des formes verbales temporelles et aspectuelles, les localisations temporelles adverbiales, l'Aktionsart des prédicats représentés par les verbes, les connaissances du monde, la relation hyponymique, les anaphores pronominales et lexicales, et la cohésion lexicale. C'est l'interaction entre plusieurs de ces indices qui fonde le choix de telle RC plutôt que (ou parfois en plus de) telle autre dans une situation donnée, et qui permet de rattacher les propositions ainsi dégagées à l'une ou l'autre des propositions contextuelles, dans une fonction « satellite » ou bien « noyau ».

\section{Les unités de discours impliquées dans les Relations de Cohérence}

D’après Mann et Thompson (1988: 248), les unités qui sont impliquées dans les schémas RST correspondent à des propositions syntaxiques («clauses») dans les textes. Il est clair qu'en général, des unités phrastiques correspondant à des clauses représentent des unités au plan du discours. Cependant, toute clause n'évoque pas nécessairement une seule proposition (cf. Asher et Lascarides, 2003; Blakemore, 2002), et il y a à l'évidence également des propositions tacites, inférées, dans tout discours (voir par exemple les «Generalized » et « Particularized Conversational Implicatures » de Levinson, 2000, dans son approche néo-gricéenne, de même que les «relational propositions » de Mann et Thompson, 1986). Berrendonner (1983), Roulet (2002) et d'autres soutiennent que les 
connecteurs relient les clauses qu'ils introduisent, non pas à la clause précédente en tant que telle, mais à l'interprétation de cette clause en fonction de son contexte (donc, à la mise en " mémoire discursive » de l'interprétation de cette clause). ${ }^{17}$ Ceci se voit de façon évidente dans les enchaînements à partir de situations (ou plus exactement, des représentations mentales que s'en sera fait le locuteur ou le scripteur) se déroulant dans le contexte d'énonciation, mais non verbalisées explicitement :

(5) [Muriel entre soudain dans le salon, où son amie Marthe est en train de lire un magazine. Marthe, surprise par le teint vert pâle des cheveux de Muriel, miironique :]

«...Alors, tu es allée chez le coiffeur?»

(6) [Une grand'mère, ouvrant sa porte d'entrée et apercevant un grand bouquet de fleurs sur le pas de la porte, le jour de ses 80 ans :]

«...Mais il ne fallait pas, voyons!»

Nous dirons la même chose pour ce qui concerne l'interprétation des anaphoriques de discours : voir comme exemple le SN défini dans le SP du bateau en (4), qui reprend un référent implicitement introduit dans le discours en amont via l'interprétation du segment lors d'une traversée maritime qui le précède. Cependant, Blakemore (2002:166-7) nie que les connecteurs dans des exemples comme ceux de (5) et (6) s'enchaînent sur une unité de discours évoquée (comme ici) implicitement, en fonction d'une inférence du locuteur à partir de la situation d'énonciation. Mais dans la conception du discours qui est celle de cet article, ceci est parfaitement légitime. D'ailleurs, c'est exactement cette situation qui prévaut dans le cas de l'emploi «exophorique » de marqueurs indexicaux (pronoms ou SN définis, démonstratifs, etc.), ce qui ne fait que renforcer la parenté de fonctionnement des connecteurs et des marqueurs indexicaux.

L'entreprise de Mann et Thompson semblerait revenir à mettre en place une sorte de "grammaire des enchaînements textuels entre clauses ", qu'elles soient explicitement reliées par un connecteur ou non. Témoin leur affirmation (1988: 244): «First, RST provides a general way to describe the relations among clauses in a text, whether or not they are grammatically or lexically signalled. " Il y a confusion cependant dans leur démarche, car d'une part, les définitions des relations de cohérence qu'ils retiennent sont formulées en termes de discours (tel que j'ai conçu ce terme, voir le Tableau 1, c'est-à-dire figurant le résultat de l'interprétation d'un segment de texte, en fonction d'un contexte approprié); mais en pratique, d'autre part, les représentations qu'ils donnent de la «structure rhétorique» des textes impliquent des relations, non directement entre propositions ou entre actes discursifs réalisés par le scripteur, mais entre segments de texte. C'est également la démarche adoptée par Roulet et al. (2001).

Mann \& Thompson excluent comme unités de discours (ou "de texte", dans mon approche) indépendantes les sujets phrastiques et les relatives restrictives, comme on l'a vu. Ceci est justifié au niveau des sujets phrastiques, car ils ont pour la plupart comme référent des propositions ou des faits (Smith, $2003:$ 88), et ne font pas avancer le temps de narration (ceci est également le fait des compléments propositionnels, selon Smith, p. 104). Selon Smith (p. 105), cependant, les relatives, même restrictives, peuvent bien faire avancer le temps de narration, tout comme les propositions « condensées » adjointes (adverbiales). Pourtant, je dirais que ce ne sont pas des unités indépendantes de discours, dans la mesure où leur rôle est de déterminer un référent pour le SN global dans lequel elles sont enchâssées. Roulet (2002: 144) souligne le bien-fondé de cette position en faisant valoir que les relatives restrictives et les complétives sujet ou complément ne 
correspondent pas, praxéologiquement, à des actes de discours, qui de ce fait seraient susceptibles d'opérer sur la mémoire discursive en construction.

Or, comme Mann et Thompson ne semblent tenir compte dans leurs analyses que du plan textuel (tout en laissant entendre, dans leurs définitions des RC ainsi que dans leurs analyses diagrammatiques, surtout, l'existence d'un plan cognitif, propositionnel et métadiscursif), il est difficile dans ce modèle d'en tenir compte de façon explicite et systématique. Nous avons vu au cours de la discussion qu'il y a des unités de niveau supérieur aux (propositions inférables à partir des) clauses: des structures composées d'un noyau et d'un ou de plusieurs satellites dépendant de ce noyau, satellites qui peuvent eux-mêmes être composés d'un noyau étendu par un ou plusieurs satellites, ainsi que de plusieurs noyaux en relation de coordination, dont un ou plusieurs peuvent à leur tour être étendu(s) par des satellites. Souvent, les satellites correspondent à des phrases indépendantes, et non à des propositions coordonnées ou subordonnées (enchâssées donc au sein d'une clause complexe ou composée). Ceci est illustré par les quatre premiers exemples présentés jusqu'à ce point.

De même, une unité de discours peut correspondre à un épisode (une unité de discours caractérisée par un seul intervalle de temps, par une unité de lieu ainsi que de thème). Ceci se voit dans la possibilité de sa reprise par un démonstratif (cf. les «abstract objects » de Asher et Lascarides, 2003).

Pour Berrendonner (2002), Roulet (2002) et d'autres, une unité de discours peut correspondre au minimum à un syntagme au plan textuel. Du point de vue du discours, la plus petite unité est représentée par un acte de discours (cf. aussi Kroon, 1999, dans le cadre de la Grammaire Fonctionnelle néerlandaise). ${ }^{18}$ Pour ces auteurs, un acte de discours est caractérisé par un passage en mémoire discursive. Voici deux exemples donnés par Roulet (2002 : 145), illustrant des actes de discours minimaux, réalisés au plan textuel par des syntagmes :

(7) (Quant à) Ma voisine, elle ne sort jamais.

(8) Malgré la pluie, elle n'hésite pas à sortir.

Le SN thématisé (en italiques) dans (7) est considéré correspondre à un acte, car il s'agit d'une mise en mémoire (ou d'un rappel thématisant) de l'entité à propos de laquelle la clause qui la suit va apporter une information. Et le SP initial italisé dans (8) sert à effectuer un contre-argument par rapport à l'acte accompli via la principale suivante.

Il y a donc différents types d'unités de discours, caractérisés chacun par le rang auquel il se situe. Ceci est le reflet du fait que le discours a un caractère structuré et hiérarchisé, à la différence du texte, qui est essentiellement linéaire (voir le Tableau 1).

\subsection{La distinction « noyau » / « satellite »}

En ce qui concerne la différenciation entre unités de discours indépendantes (" noyaux » dans la terminologie RST) et dépendantes ("satellites»), nous avons déjà indiqué que la présence d'un ou de plusieurs anaphoriques dans une unité signale sa dépendance vis-àvis d'une unité dont elle dépend (qui permet donc en principe la résolution du ou des anaphorique(s)). D'autres indices pourront être la présence de connecteurs devant une unité, ou bien la possibilité d'en insérer, tout en gardant le même type de relation entre les propositions concernées. En général, cet indice signale la dépendance de l'unité qui suit le connecteur par rapport à celle qui la précède - mais ceci n'est pas toujours le cas (voir l'interprétation concessive de la conjonction but dans l'exemple (2)). Un autre indice 
du statut de satellite serait le type de temps verbal utilisé par le verbe de la principale : le plus-que-parfait, comme on l'a vu, ainsi que l'imparfait, indiquent une relation d'arrièreplan par rapport à l'unité précédente, et ne font pas avancer le temps de la narration (dans le cas des segments de texte narratifs). Plus généralement, selon Mann et Thompson (1988: 266), on peut analyser les unités de discours en noyau et satellite du point de vue de leurs fonctions respectives dans le discours (je traduis) :

- Il arrive souvent qu'un membre de la paire de segments soit incompréhensible indépendamment de l'autre, mais pas vice versa. Sans l'assertion du noyau, le satellite « évidence » constitue un illogisme, tout comme le satellite d'arrière-plan sans l'empan noyau qu'il contextualise.

- Souvent également, un membre de la paire est plus susceptible de substitution que l'autre. Un satellite de type Evidence peut être remplacé par une preuve totalement différente, sans que la fonction du texte dans son ensemble en soit beaucoup modifiée. Par contre, le remplacement d'une assertion (« claim ») aura des effets bien plus drastiques.

- Il arrive aussi que l'un des membres de la paire soit plus essentiel à l'objectif visé par le scripteur que l'autre.

\section{Conclusion}

Les moyens susceptibles d'assurer la cohésion textuelle (relations entre temps des verbes, expressions adverbiales temporelles ou spatiales et leur positionnement dans le texte, relations lexicales entre prédicateurs, relations anaphoriques, fonctionnement des connecteurs, articulation syntaxique des propositions) ne sauraient se caractériser sans la prise en compte de la cohérence du discours qui leur permet d'assumer leur rôle cohésif contrairement à ce que laissent entendre Halliday et Hasan (1976) (cf. aussi la critique de Brown et Yule, 1983); et parallèlement, les relations de cohérence ou de discours au moyen desquelles les unités discursives pourront se dégager et s'intégrer pour former une unité de niveau supérieur, ne peuvent se caractériser et se reconnaitre sans la prise en compte du fonctionnement des moyens cohésifs - contradictoirement au mode de caractérisation de ces relations employé par Mann et Thompson (1988). D'après les quelques textes courts que nous avons analysés ci-dessus, ${ }^{19}$ il est clair que ces deux dimensions de la structure des textes sont complémentaires, voire interdépendantes. En fait, texte, contexte et discours sont interdéfinissables.

Quant à Hobbs (1990), à part l'heuristique que fournissent certains types de connecteurs de discours, il n'exploite pas les indices apportés par de tels facteurs formels et sémantiques en distinguant entre les différents types de relation de cohérence qu'il propose; plutôt, il les invoque quasi-exclusivement en terme de critères basés sur la connaissance ou la cognition, critères qui conduiraient à l'induction d'inférences au moyen desquelles le lecteur ferait sens du texte. Dans la RST classique de Mann et Thompson, semblablement, aucun recours n'est fait aux indices co-textuels ou contextuels, qui permettraient d'étayer la reconnaissance de telle ou telle de leurs relations dans un contexte donné. Cette absence d'enracinement empirique fait qu'il est difficile de distinguer entre la pertinence de telle relation plutôt que telle autre pour intégrer deux unités dans ce cadre. Nous avons éprouvé cette difficulté en ré-analysant certains des exemples qu'ils donnent eux-mêmes (textes (1)-(3) ci-dessus).

Comme le discours, au sens où je l'entends (voir le Tableau 1), est toujours provisoire et est construit dynamiquement par les interlocuteurs (ou par scripteur et lecteur), la 
possibilité de ré-analyse de tel ou tel segment de texte en amont, suite à une difficulté d'intégration d'une unité de discours qui vient d'être construite, est réelle : elle devrait donc être prise en compte par tout modèle de la création du discours à partir d'un texte et d'un contexte pertinent. Nous avons vu un exemple patent de la nécessité d'une telle ré-analyse dans le cas de l'exemple (2) donné par Mann et Thompson (1988).

Liée à cette possibilité de ré-analyse et de révision de la construction d'une unité supérieure de discours qui viendrait de se faire, est la possibilité de superposition, dans l'intégration de deux unités, de plus d'une relation de cohérence. ${ }^{20}$ Cette possibilité est explicitement exclue par Mann et Thompson, mais est admise par Hobbs et par Asher \& Lascarides. Nous venons d'en voir la réalité en analysant les textes mêmes de Mann et Thompson (voir le texte (3) pour une illustration, ainsi que le texte (4) proposé par moimême).

Enfin, en ce qui concerne la question des unités de discours qu'il conviendrait de reconnaître, nous avons vu qu'il y a d'autres unités pertinentes en plus de celle (censée être minimale) reconnue à la fois par Mann et Thompson et par Hobbs, évoquée via la proposition syntaxique («clause »): par exemple, celles qui seraient exprimées par les nominalisées (voir la seconde unité minimale dans le texte (4)) ou par les syntagmes prépositionnels ou nominaux qui correspondent praxéologiquement à un acte de discours (cf. Roulet, 2002 : exemples (7) et (8) ci-dessus); puis les unités de rang supérieur formées suite à l'intégration de deux (ou de plus de deux) unités plus petites.

\section{BIBLIOGRAPHIE}

Asher, N. \& Lascarides, A. (2003). Logics of Conversation. Cambridge: Cambridge University Press.

Berrendonner, A. (1983). Connecteurs pragmatiques et anaphore. Cahiers de Linguistique Française $5: 215-246$.

Berrendonner, A. (2002). Morpho-syntaxe, pragma-syntaxe et ambivalences sémantiques. In H. L. Andersen \& H. Nølke (éds), Macro-syntaxe et macro-sémantique. Actes du colloque d'Arhus, 17-19 mai 2001, Berne : Peter Lang, 23-41.

Blakemore, D. (2002). Relevance and Linguistic Meaning. The semantics and pragmatics of discourse markers. Cambridge: Cambridge University Press.

Brown, G. \& Yule, G. (1983). Discourse Analysis. Cambridge: Cambridge University Press.

Butler, C.S. (2003). Structure and Function. A guide to three major structural-functional theories. Part 2: From clause to discourse and beyond. Amsterdam \& Philadelphia: John Benjamins.

Cornish, F. (2003a). Types de relations de discours entre énoncés : interactions avec l'anaphore transphrastique, Cahiers du CRISCO (Université de Caen) $12: 69-84$.

Cornish, F. (2003b). The roles of (written) text and anaphor-type distribution in the construction of discourse. Text 23(1): 1-26. 
Cornish, F. (2006). Relations de cohérence et anaphores en contexte inter-phrastique : une symbiose parfaite. À paraître dans un numéro spécial de la revue Langages, $\mathrm{n}^{\circ} 164$, intitulé Unité(s) du texte (coord. D. Legallois).

Coupland, N., Wiemann, J.M. \& Giles, H. (1991). Talk as "problem" and communication as "miscommunication"; an integrative analysis. Ch. 1 in Coupland, N., Giles, H. \& Wiemann, J.M. (éds.) "Miscommunication" and Problematic Talk. London \& New Delhi: Sage Publications, 1-17.

Fabricius-Hansen, C., \& Behrens B. (2001). Elaboration and related discourse relations viewed from an interlingual perspective. SPRIK report of the project Languages in Contrast, University of Oslo. (Disponible à http://www.hf.uio.no/german/sprik).

Gumperz, J. J. (1992). Contextualization and understanding. Ch. 8 in Duranti, A. \& Goodwin, C. (éds), Rethinking context. Language as an interactive phenomenon, Cambridge: Cambridge University Press, 229-252.

Halliday, M. A.K. \& Hasan, R. (1976). Cohesion in English. London: Longman.

Hobbs, J. R. (1990). Ch. 5: The coherence and structure of discourse. In Literature and Cognition, Leland Stanford Junior University, Calif: CSLI Lecture Notes 21, 83-114.

Knott, A. \& Sanders, T. (1998). The classification of coherence relations and their linguistic markers: an exploration of two languages. Journal of Pragmatics 30: 135-175.

Kroon, C. (1999). Discourse markers, discourse structure and Functional Grammar. In C. S. Butler, J. H. Connolly, R. A. Gatward \& R. M. Vismans (éds.) Discourse and Pragmatics in Functional Grammar. Berlin/New York: Mouton de Gruyter, 17-32.

Legallois, D. (2006). Quand le texte signale sa structure : la fonction textuelle des noms sous spécifiés. Ce numéro.

Levinson, S. (2000). Presumptive Meanings. The theory of Generalized Conversational Implicature. Cambridge, Mass.: MIT Press.

Mann, W. C. \& Thompson, S. A. (1986). Relational propositions in discourse. Discourse Processes 9 : 57-90.

Mann, W. C. \& Thompson, S. A. (1988). Rhetorical Structure Theory: Toward a functional theory of text organization. Text 8(3): 243-281.

Marcu, D. (2000). The rhetorical parsing of unrestricted texts: A surface-based approach. Computational Linguistics 26(3): 395-448.

Parisi, D. \& Castelfranchi, C. (1977). The discourse as a hierarchy of goals. Signs of Change 1(2): 31-67.

Rossari, C. (2000). Connecteurs et relations de discours : des liens entre cognition et signification. Nancy : Presses Universitaires de Nancy.

Rossari, C., Beaulieu-Masson, A., Cojocariu, C. \& Razgouliaeva, A. (2004). Autour des connecteurs. Berne : Peter Lang.

Roulet, E. (2002). Ch. VII : De la nécessité de distinguer des relations de discours sémantiques, textuelles et praxéologiques. In H. L. Andersen \& H. Nølke (éds.) Macro-syntaxe et macro-sémantique . Actes du colloque d'Arhus, 17-19 mai 2001, Berne : Peter Lang, 141-165.

Roulet, E., Filliettaz, L. \& Grobet, A. (2001). Un modèle et un instrument d'analyse de l'organisation du discours. Berne: Peter Lang. 
Sanders, T.J.M. (1997). Semantic and pragmatic sources of coherence: on the categorisation of coherence relations in context. Discourse Processes 24: 119-147.

Smith, C. S. (2003). Modes of Discourse. The local structure of texts. Cambridge: Cambridge University Press.

Webber, B., Joshi, A., Stone, M. \& Knott, A. (2003). Anaphora and discourse structure. Computational Linguistics 29(4): 545-587.

Widdowson, H.G. (2004). Text, context, pretext. Critical issues in Discourse Analysis. Oxford : Blackwell.

\section{NOTES}

1. Sous le titre Relations de Cohérence et Fonctionnement des Anaphores, projet commencé au mois d'avril 2003.

2. Nous avons retenu dans notre projet ILF une soixantaine de textes de ce genre, ainsi qu'une centaine d'autres articles ou extraits d'articles sous forme électronique du journal Le Monde.

3. Comme si cela ne pouvait jamais être le cas!

4. Un peu comme les conditions proposées par J.R. Searle pour l'existence des types d'illocution et de perlocution.

5. Elle est mentionnée en Appendice à la p. 277 comme encore à formuler.

6. En tout cas, en ce qui concerne le risque que la substance en question poserait aux humains.

7. Voir les définitions de la relation Elaboration supra.

8. Voir les définitions de la relation Evidence infra.

9. Voir Fabricius-Hansen \& Behrens (2001) pour une discussion intéressante de cette relation, basée sur des données translangagières.

10. Qui admet néanmoins que plusieurs analyses alternatives puissent s'appliquer à une paire d'unités donnée.

11. Ceux que les auteurs donnent eux-mêmes en exemples.

12. Mais voir Marcu (2000) pour une tentative récente de dériver automatiquement des structures textuelles RST, principalement par le biais des expressions-signaux («cue-phrases») (connecteurs ainsi qu'expressions adverbiales) figurant dans telle ou telle position dans les textes analysés. Webber et al. (2003), pour leur part, proposent de faire une distinction de principe entre « connecteurs structuraux» (because, but, so etc.) et "adverbiaux de discours » (then, otherwise, nevertheless), dont le sens instructionnel implique une composante anaphorique. Cependant, ce dernier article ne relève pas du cadre de la RST.

13. Qui contient une référence aux arbres, référent qui de ce fait assume le statut de topique de ce segment de discours dans son ensemble.

14. Ceci est bien entendu la raison pour laquelle Mann et Thompson (1988) avaient rejeté le recours aux indices « textuels » dans leurs définitions de telles relations.

15. Chaque unité minimale dégagée du texte (une proposition finie ou non-finie, coordonnée ou subordonnée, ou bien nominalisée, dans mon approche) est annotée pour sa structure événementielle. J'emploie pour cela la notation proposée par Smith (2003) (mentions en indice «EV » pour «événement» ou «ET » pour «état»). Les symboles préfixés ' $\mathrm{S}^{\circ}$ ', ' $\mathrm{S}$ ', etc. sont employés pour représenter les unités minimales du texte, suivant Hobbs (1990).

16. Les traitements infligés au XVIIIème siècle étant connus comme étant beaucoup plus draconiens que ceux en vigueur aujourd'hui.

17. Voir Rossari (2000) et Rossari et al. (2004) pour des analyses en profondeur d'une gamme de connecteurs. 
18. La plus petite unité textuelle, elle, correspondrait en fait à un seul morphème : par exemple, Non! en réponse à une question ou à une requête, ou une salutation de la main en réaction au Bonjour ! du premier locuteur. Je remercie Anne Grobet de m'avoir rappelé ce point.

19. Voir aussi Cornish (à paraître, 2006) pour d'autres du même genre.

20. À condition, bien entendu, que ces relations ne soient pas en contradiction l'une avec l'autre.

\section{RÉSUMÉS}

L'article porte principalement sur les critères et les indices de reconnaissance d'un sousensemble de relations de cohérence (ou de discours), en fonction d'une sélection de courts textes attestés en anglais et en français - souvent sans connecteur explicite. Est également abordée la question de la nature et des types d'unités de discours impliquées dans l'intégration grâce aux relations de cohérence, et de leur point d'attachement. L'article soutient que, tout comme les relations de cohérence ne peuvent guère être identifiées sans la prise en compte d'un ensemble d'indices de cohésion textuels, de même le fonctionnement de ces derniers ne saura être pleinement saisi en l'absence de leur rôle dans la mise en place d'une (ou à l'occasion, de plus d'une) relation de cohérence discursive. Cohésion textuelle et cohérence discursive sont donc complémentaires, voire interdépendantes. Une critique de l'approche de la RST de W.C. Mann et S.A. Thompson de telles relations forme la base de la discussion.

The article deals mainly with the criteria and heuristic cues for a subset of coherence (or discourse) relations, in terms of a selection of short attested English and French texts - often without explicit connectives. The issue of the nature and types of discourse units involved in the integration via given coherence relations, and of their point of attachment, is also discussed. The article argues that, just as coherence relations can only be identified by exploiting a range of cues to textual cohesion, so the functioning of the latter can only be fully grasped by taking account of the role they play in the setting up of a discourse coherence relation (or on occasion, of more than one such relation). Textual cohesion and discourse coherence are thus complementary, and even interdependent. A critique of W.C. Mann and S.A. Thompson's RST approach to such relations forms the basis for the discussion.

\section{INDEX}

Keywords : coherence, cohesion, context, discourse, discourse units

Mots-clés : cohérence, cohésion, discours, unités de discours, contexte

\section{AUTEUR}

FRANCIS CORNISH

ERSS, CNRS UMR 5610 et Université de Toulouse-Le Mirail 